\title{
Niaouli Oil
}

National Cancer Institute

\section{Source}

National Cancer Institute. Niaouli Oil. NCI Thesaurus. Code C72212.

The essential oil extracted from the foliage and twigs of Melaleuca viridiflora. Niaouli oil is used for aromatherapy and for its antiseptic properties. 